\title{
Editorials
}

\section{Severe sepsis: a bundle still under construction?}

Robert N. Sladen MB ChB MRCP(UK) FRCP(C)

W HAT is "severe sepsis"? Perhaps it is worthwhile reiterating the consensus reached regarding sepsis nomenclature in $1992^{1}$ because it provides the framework for any discussion of evidence-based intervention. The term systemic inflammatory response syndrome (SIRS) emphasizes that the body's response to infection may be more damaging than the invading organism itself. Systemic inflammatory response syndrome is defined by its clinical manifestations, including fever $\left(\geq 38^{\circ} \mathrm{C}\right)$ or hypothermia $\left(\leq 36^{\circ} \mathrm{C}\right)$; tachycardia $(>90$ beats. $\left.\mathrm{min}^{-1}\right)$; tachypnea ( $>20$ breaths. $\mathrm{min}^{-1}$ ) or hypocarbia $\left(\mathrm{PaCO}_{2}<32 \mathrm{mmHg}\right)$; and leukocytosis (> 12 $\left.\mathrm{k} \cdot \mathrm{mm}^{3}\right)$, leukopenia $\left(<4 \mathrm{k} \cdot \mathrm{mm}^{3}\right)$, or an elevated band count (>10\%). Sepsis is present when the manifestations of SIRS are associated with an infection. Severe sepsis is present when sepsis-induced hypoperfusion induces end-organ dysfunction (e.g., renal dysfunction, acute respiratory failure). Septic shock is present when hypotension persists despite fluid resuscitation, with manifestations of tissue hypoperfusion such as elevated arterial lactate. Hypotension is defined as a systolic blood pressure $(\mathrm{SBP})<90 \mathrm{mmHg}$, mean arterial blood pressure $<65 \mathrm{mmHg}$, or a decrease in SBP by more than $40 \mathrm{mmHg}$.

Although the last two decades have seen remarkable progress in its diagnosis and management, sepsis remains a formidable foe. In 2001, Angus et al. ${ }^{2}$ estimated that three-quarters of a million cases of severe sepsis occur in the USA each year, about three cases per 1,000 population. Moreover, severe sepsis has a mortality of nearly $30 \%$, represents nearly $10 \%$ of all US deaths (equivalent to acute myocardial infarction) and imparts an annual cost burden of more than 16 billion US dollars.

This compelling condition has galvanized an international collaboration called the Surviving Sepsis
Campaign, developed "to improve the management, diagnosis, and treatment of sepsis" by the European Society of Intensive Care Medicine, the International Sepsis Forum, and the Society of Critical Care Medicine (http://ssc.sccm.org/). On its website, the Surviving Sepsis Campaign provides guidelines to resuscitation and management in severe sepsis grouped into therapeutic packages called bundles. The term bundle is explained as "a group of interventions related to a disease process that, when executed together, result in better outcomes than when implemented individually. The individual bundle elements are built upon evidence-based practices. The science behind the elements of a bundle is so well-established that their implementation should be considered a generally accepted practice (my italics)". This definition appears to brook little further discussion!

In 2006, an editorial in the New England Journal of Medicine ${ }^{3}$ criticized the Surviving Sepsis Campaign because of its funding support by Eli Lilly, the manufacturer of human recombinant activated protein $\mathrm{C}$ (drotrecogin), an integral component of the severe sepsis bundle. The editorial also opined that guidelines based on evidence-based medicine favour new over standard interventions. For example, drotrecogin ... "was given a highly favourable rating (grade B), whereas established therapies for sepsis (such as antibiotics, fluids, and vasopressors), though included in the recommendations, received lower ratings (grade $\mathrm{D}$ or E), because most had not undergone randomized, controlled trials owing to a lack of equipoise".

Perhaps in the spirit of challenging this assumption, in this issue of the Journal the Canadian Critical Care Trials Group present their observations on the influence of the quantity and type of fluid on resuscitation of patients with early severe sepsis. ${ }^{4}$ Their task is somewhat daunting in that multiple trials of resuscitation

CAN J ANESTH 2007 / 54: $10 /$ pp 779-785

From the Department of Anesthesiology, College of Physicians and Surgeons of Columbia University, New York, New York, USA. Address correspondence to: Dr. Robert N. Sladen, Department of Anesthesiology PH 527-B, College of Physicians and Surgeons of Columbia University, 630 W. 168 ${ }^{\text {th }}$ St, New York, NY 10032 USA. Phone: 212-305-8633; E-mail: rs543@columbia.edu 
over many years have shown no significant outcome difference between colloid or crystalloid-based resuscitation. In 2004 the Australian and New Zealand Intensive Care Society Clinical Trials Group published a landmark randomized controlled trial, the SAFE (Saline vs Albumin Fluid Evaluation) Study. ${ }^{5}$ In nearly 7,000 critically ill patients, there was no difference in outcome between the use of $4 \%$ human albumin solution and normal saline. Of note, the Surviving Sepsis Campaign Resuscitation Bundle mandates an initial fluid challenge with $20 \mathrm{~mL} \cdot \mathrm{kg}^{-1}$ of crystalloid, but also states "a colloid equivalent is an acceptable alternative to crystalloid". This is followed by the caveat that "....as the volume of distribution is much larger for crystalloids than for colloids, resuscitation with crystalloids requires more fluid to achieve the same goals and results in more edema" (http://ssc.sccm.org/).

Apparently undeterred, McIntyre et al. ${ }^{4}$ and the Canadian Critical Care Trials Group set out to examine quantity and type of fluid administered in the first six hours after the identification of severe sepsis, and its association with hospital and intensive care unit (ICU) mortality, and organ system failure. They appear to have been inspired by another landmark study, published in 2001 by Rivers et al. ${ }^{6}$ In patients with severe sepsis, early ( $<$ six hours), goal-directed therapy in the emergency room decreased subsequent in-hospital mortality from 46.5 to $30.5 \%$.

McIntyre et al. ${ }^{4}$ point out that the Rivers study did not address the question of which fluid would best achieve the goals that might decrease mortality. They therefore devised a retrospective, multicentre cohort study in five Canadian tertiary care ICUs, obtaining data on patients with severe sepsis over a two-year period from 2000-2002. They recorded the total quantity of fluid administered in the six hours after the first episode of hypotension, and categorized it as $0-2$ $\mathrm{L}, 2-4 \mathrm{~L}$ and $>4 \mathrm{~L}$. They grouped the type of fluid administered as crystalloid alone or a combination of colloid and crystalloid, and also included blood products into the fluid volume. The outcomes included hospital mortality, ICU mortality and organ system failure within $24 \mathrm{hr}$. The latter was assessed by the Sequential Organ Failure Assessment (SOFA) score. ${ }^{7}$ The SOFA score defines organ failure in six systems by a single criterion from each: hypotension, $\mathrm{PaO}_{2}$ to $\mathrm{FIO}_{2}$ ratio, bilirubin, creatinine, Glasgow Coma Score and platelet count. These are scored from 0 to 4 , from normal function to severe organ failure. In the present study the authors defined individual organ failure as a SOFA score of $\geq 3$.

McIntyre et al. ${ }^{4}$ examined more than 2,000 charts to find 496 patients that met their eligibility criteria.
Using multivariable regression analysis, they were unable to detect any statistically significant association between the quantity or type of fluid administered in the first six hours after the diagnosis of severe sepsis, and hospital and ICU mortality or organ failure. They did find an increased risk of cardiovascular failure and decreased risk of renal failure when more fluid was given. Moreover, they found that administration of colloid and crystalloid fluid was associated with a lower risk of renal failure than when crystalloid alone was used.

What impact should these findings have on our clinical management of patients with severe sepsis? The authors might be the first to agree that at present the answer is - none. Their study is a retrospective review and was not hypothesis-driven, and there was no analysis that could have predicted whether their survey was sufficiently powered to detect an outcome difference. The authors themselves conclude that their findings "... should be considered as hypothesis-generating and warrant confirmation or refutation by randomized controlled trials". But the question is - what hypotheses are generated by their findings?

The first hypothesis could be that a sufficient volume of fluid administered at the first sign of hypotension might alter the outcome of severe sepsis. McIntyre et al. quantified the volume of fluid administered to their patients in the first six hours as $0-2$ $\mathrm{L}, 2-4 \mathrm{~L}$ and $>4 \mathrm{~L}$. But we have no idea of knowing why their patients were given a small, intermediate or large amount of fluid. Was it based on hemodynamic findings? Institutional protocol? Common practice? In the Rivers study ${ }^{6}$ that the authors used as a model, the emphasis was not on the volume or type of fluid used, but on goal directed therapy, defined as achieving reversal of hypotension, or a central venous oxygen saturation of $65 \%$. This approach is also advocated in the Sepsis Resuscitation Bundle of the Surviving Sepsis Campaign (http://ssc.sccm.org/).

In the McIntyre study ${ }^{4}$ the average total amount of fluid administered was only $2.4 \mathrm{~L}$, compared to an average of $5.0 \mathrm{~L}$ in the Rivers study. ${ }^{6}$ Could this be accounted for by the application of goal directed therapy in the latter? Because McIntyre et al. ${ }^{4}$ were unable to get details of the rate of fluid administration or goal directed therapy, their study is unable to shed any light on the relationship between the volume of fluid administered, achievement of therapeutic goals, and outcome.

The second hypothesis could be that a combination of colloid and crystalloid might have a more beneficial affect on outcome than crystalloid alone. But, as McIntyre et al. ${ }^{4}$ themselves acknowledge, an excellent 
hypothesis-generating study has already been done: in the SAFE study, there was a trend towards lower mortality in a severe sepsis subgroup that received albumin rather than normal saline. ${ }^{5}$ It seems unfortunate that the Canadian Critical Care Trials Group did not choose to test this hypothesis with a randomized controlled trial - perhaps they will do so now?

There are several other unanswered questions. McIntyre et al. ${ }^{4}$ excluded patients who developed severe sepsis after the first $24 \mathrm{hr}$ following ICU admission or after seven days of hospitalization. This might be an important subgroup, especially for sepsis developing in post-surgical patients, which comprised only $25 \%$ of their study. The Canadian Critical Care Trials Group should examine their data base to ascertain what proportion this represents of the total number of patients with severe sepsis, and whether they have a significant difference in outcome. The Rivers trial was based on early intervention in patients admitted to an emergency room. ${ }^{6}$ Ultimately, we need to know whether the Rivers criteria (and the Sepsis Resuscitation Bundle of the Surviving Sepsis Campaign) are equally effective in treating patients who develop severe sepsis after the first $24 \mathrm{hr}$ following ICU admission or after seven days of hospitalization.

What do we make of the authors' observations that an increased volume of fluid administered was associated with increased cardiovascular but decreased renal failure? One would be tempted to conclude, "What is bad for the heart is good for the kidneys". But was renal function preserved in the same patients in whom increased fluid also caused cardiac failure? Were the patients who developed cardiac failure simply given excessive fluid? Or could it be that patients requiring more fluid are sicker, with a more severe capillary leak syndrome and septic myocardial depression to begin with? Certainly these are all hypotheses worth testing, but may be difficult - or even unethical - to build into a randomized controlled trial.

Perhaps the most intriguing observation is that a combination of colloid and crystalloid was associated with decreased renal failure. ${ }^{4}$ Could this be supportive evidence that hemodynamic resuscitation is better accomplished by the addition of colloid? One of the problems in simply comparing volumes of fluid is that the intravascular volume expansion effect is quite different between crystalloid and colloid. In the SAFE study, ${ }^{5}$ the volume of normal saline deemed equivalent to $4 \%$ albumin was 1.3 to $1.6: 1$. In anesthesiology and surgery circles, there are some strong advocates for intraoperative fluid restriction during gastrointestinal surgery because of evidence of better postoperative outcomes. ${ }^{8}$ When one examines the studies, however, one finds that a "fluid restricted" approach simply substitutes colloid for crystalloid, thus decreasing the total volume administered while maintaining the hemodynamic benefit. Thus, it is conceivable that in the present study patients had better renal outcome with colloid and crystalloid, because the former achieves better volume expansion than crystalloid alone.

At the end, what have we learned from this observational study? Certainly, it reinforces the forbidding implications of severe sepsis. More than a third of patients suffered two or more organ failures within 24 hr of its onset; ICU mortality was nearly $35 \%$, hospital mortality over $45 \%$, and median ICU and hospital length of stay six and 14 days respectively.

More pertinently, as the authors themselves conclude, we need to generate more evidence on the specifics of early fluid management in severe sepsis. There appears to be considerable merit in testing the hypothesis that in severe sepsis, early resuscitation with a combination of colloid and crystalloid is better for outcome (and renal preservation) than crystalloid alone. But any such study must incorporate goal directed therapy - and achievement of those goals - as the primary yardstick of management, rather than the total volume of fluid used.

\section{La septicémie sévère : un lot encore en travaux?}

Qu'est-ce qu'une "septicémie sévère " ? Il peut être utile de rappeler le consensus atteint en 1992 concernant la nomenclature de la septicémie, ${ }^{1}$ car il fournit un cadre à toute discussion portant sur une intervention basée sur des données probantes. Le terme syndrome de réaction inflammatoire systémique (SIRS) souligne le fait que la réaction du corps à une infection peut provoquer davantage de dégâts que l'organisme envahisseur en soi. Le syndrome de réaction inflammatoire systémique se définit par ses manifestations cliniques : fièvre $\left(\geq 38^{\circ} \mathrm{C}\right)$ ou hypothermie $\left(\leq 36^{\circ} \mathrm{C}\right)$; tachycardie $\left(>90\right.$ battements. $\left.\mathrm{min}^{-1}\right)$; tachypnée (> 20 respirations $\cdot \mathrm{min}^{-1}$ ) ou hypocapnie $\left(\mathrm{PaCO}_{2}<32 \mathrm{mmHg}\right)$; et hyperleucocytose (> 12 $\left.\mathrm{k} \cdot \mathrm{mm}^{3}\right)$, leucopénie $\left(<4 \mathrm{k} \cdot \mathrm{mm}^{3}\right)$, ou un décompte de bandelettes élevé (> $10 \%$ ). On parle de septicémie 
lorsque les manifestations du SIRS sont associées à une infection. Il est question de septicémie sévère lorsque l'hypoperfusion provoquée par la septicémie cause une dysfonction des organes (par ex., insuffisance rénale, insuffisance respiratoire aiguë). Enfin, on parle de choc septique lorsque l'hypotension persiste malgré une réanimation liquidienne, accompagnée de manifestations d'hypoperfusion tissulaire, comme par ex. un lactate artériel élevé. L'hypotension est définie en tant qu'une pression systolique $(\mathrm{SBP})<90 \mathrm{mmHg}$, une pression artérielle moyenne $<65 \mathrm{mmHg}$, ou une diminution de la SBP de plus de $40 \mathrm{mmHg}$.

Bien que nous ayons assisté, durant les vingt dernières années, à des progrès considérables dans le diagnostic et la prise en charge de la septicémie, celleci demeure néanmoins un ennemi redoutable. En 2001, Angus et coll. ${ }^{2}$ ont estimé que 750000 cas de septicémie sévère surviennent chaque année aux EtatsUnis, c'est-à-dire environ trois cas sur mille personnes. En outre, la septicémie sévère provoque la mort dans presque $30 \%$ des cas, représente environ $10 \%$ du nombre total de décès aux Etats-Unis (similaire au taux pour l'infarctus du myocarde aigu), et coûte annuellement plus de 16 milliards USD.

Cet état de fait alarmant a stimulé une collaboration internationale nommée "Surviving Sepsis Campaign » (Campagne de survie à la septicémie), développée dans le but "d'améliorer la prise en charge, le diagnostic et le traitement de la septicémie » par la Société européenne de soins intensifs, le International Sepsis Forum et la Society of Critical Care Medicine (http://ssc.sccm.org/). Sur son site Internet, la Surviving Sepsis Campaign fournit des directives pour la réanimation et la prise en charge dans les cas de septicémie sévère; ces directives sont regroupées en paquets thérapeutiques appelés lots, ou « bundles » en anglais. Ce terme est défini comme "un groupe d'interventions associées à un processus de maladie qui, si elles sont exécutées ensemble, ont pour résultat de meilleurs devenirs que lorsqu'elles sont mises en place une à une. Les éléments individuels du lot sont fondés sur des pratiques basées sur des données probantes. $L a$ science soutenant les éléments d'un lot est tellement bien établie que leur implémentation devrait être considérée comme une pratique généralement acceptée (italiques ajoutées) ». Cette définition ne semble admettre que peu de discussion!

En 2006, un éditorial du New England Journal of Medicine ${ }^{3}$ critiquait la Surviving Sepsis Campaign à cause du soutien financier de l'initiative par Eli Lilly, le fabricant de la protéine C activée recombinante humaine (drotrécogine), une composante à part entière du lot de la septicémie sévère. L'éditorial était également d'avis que ces directives fondées sur une pratique médicale basée sur des données probantes prônaient les nouvelles méthodes d'intervention plutôt que les méthodes standard. Par exemple, la drotrécogine ... «a reçu un score très favorable (note B), alors que d'autres thérapies bien établies pour le traitement de la septicémie (comme les antibiotiques, les liquides, et les vasopresseurs), bien que comprises dans les recommandations, ont reçu des scores plus bas (note $\mathrm{D}$ ou $\mathrm{E}$ ), parce que la plupart de ces méthodes n'avaient pas fait l'objet d'études randomisées contrôlées par manque du principe d'équilibre (equipoise). »

Dans ce numéro du Journal, et peut-être en réponse à cette hypothèse, le Canadian Critical Care Trials Group présente ses observations concernant l'influence de la quantité et du type de liquide administré sur la réanimation de patients atteints de septicémie sévère précoce. ${ }^{4} \mathrm{La}$ tâche du groupe est quelque peu décourageante, étant donné que de nombreuses études traitant de la réanimation sur plusieurs années n'ont pas montré de différence de résultat significative entre la réanimation colloïde ou cristalloïde. En 2004, le Australian and New Zealand Intensive Care Society Clinical Trials Group a publié une étude randomisée contrôlée phare: l'étude SAFE (Saline vs Albumin Fluid Evaluation). ${ }^{5}$ Chez près de 7000 patients sévèrement malades, aucune différence n'a été observée dans le devenir entre l'utilisation d'albumine humaine à $4 \%$ et de solution saline normale. Il est à noter que le lot de réanimation (Resuscitation Bundle) de la Surviving Sepsis Campaign recommande une charge liquidienne d'attaque initiale de cristalloïde $20 \mathrm{~mL} \cdot \mathrm{kg}^{-1}$, mais déclare également qu'un «équivalent colloïde est une alternative acceptable au liquide cristalloïde. » S'ensuit l'avertissement que "...étant donné que le volume de distribution est bien plus vaste pour les liquides cristalloïdes que pour les colloïdes, la réanimation avec des cristalloïdes nécessite davantage de liquide pour atteindre les mêmes objectifs et résulte en plus d'œdème » (http://ssc.sccm.org/).

Apparemment, tout cela n'a pas suffi à décourager McIntyre et coll. ${ }^{4}$ ainsi que le Canadian Critical Care Trials Group, qui ont entrepris d'examiner la quantité et le type de liquide administré durant les six premières heures suivant l'identification d'une septicémie sévère, ainsi que son rapport avec la mortalité hospitalière et aux soins intensifs (ICU), et avec une défaillance systémique. Les auteurs semblent avoir été inspirés d'une autre étude jalon, publiée en 2001 par Rivers et coll. ${ }^{6}$ Des patients atteints de septicémie sévère, qui ont reçu une approche thérapeutique aux objectifs déterminés 
(goal directed therapy) précoce ( $<$ six heures) dans la salle des urgences a permis de réduire la mortalité hospitalière de 46,5 à $30,5 \%$.

McIntyre et coll. ${ }^{4}$ soulignent que l'étude Rivers ne traite pas du type de liquide, à savoir quel type de liquide atteindrait le mieux les objectifs pouvant réduire la mortalité. C'est pourquoi ils ont élaboré une étude de cohorte rétrospective multi-centrique dans cinq unités de soins intensifs canadiennes de soins tertiaires, et ont compilé les données de patients atteints de septicémie sévère durant une période de deux ans (2000 à 2002). Ils ont enregistré la quantité totale de liquide administré durant les six premières heures suivant le premier épisode d'hypotension, et l'ont classifié en 0-2 L, 2-4 L, et > 4 L. Les auteurs ont regroupé le type de liquide administré en cristalloïde seul ou combinaison de colloïde et cristalloïde, et ont également inclus les produits sanguins dans le volume de liquide. Les résultats mesurés comprenaient la mortalité hospitalière, la mortalité aux soins intensifs, et la défaillance systémique durant les premières $24 \mathrm{~h}$. Cette dernière donnée a été évaluée à l'aide du score de SOFA (Sequential Organ Failure Assessment). ${ }^{7}$ Le score de SOFA détermine la défaillance systémique dans six systèmes par un critère unique de chacun de ces systèmes : hypotension, ratio $\mathrm{PaO}_{2} / \mathrm{F}_{\mathrm{I}} \mathrm{O}_{2}$, bilirubine, créatinine, score de Glasgow (GCS) et décompte plaquettaire. Ces données reçoivent un score allant de 0 à 4 , de fonction normale à défaillance systémique sévère. Dans l'étude en question, les auteurs ont déterminé la défaillance systémique des organes individuels comme atteignant un score de SOFA $\geq 3$.

McIntyre et coll. ${ }^{4}$ ont analysé plus de 2000 rapports médicaux et ont retenu 496 patients qui répondaient à leurs critères d'éligibilité. En se basant sur des analyses de régression multivariées, les auteurs n'ont pu détecter aucune association statistiquement significative entre la quantité ou le type de liquide administré durant les six premières heures suivant le diagnostic de la septicémie sévère et la mortalité hospitalière/en soins intensifs ou la défaillance systémique. Par contre, ils ont observé un risque accru de défaillance cardiovasculaire et un risque réduit de défaillance rénale lors d'une administration plus importante de liquide. En outre, ils ont découvert que l'administration combinée de colloïde et de cristalloïde était associée à un risque réduit d'insuffisance rénale en comparaison à l'administration de cristalloïde seul.

Quel impact devraient avoir ces données sur la prise en charge clinique de patients atteints de septicémie sévère? Les auteurs seront peut-être les premiers à être d'avis que, pour le moment, la réponse est : aucun. Leur étude est un compte-rendu rétrospectif et n'était pas basée sur une hypothèse; en outre, aucune analyse n'aurait pu prévoir si leur étude avait une portée suffisante à détecter une différence dans les résultats. Les auteurs eux-mêmes concluent que leurs résultats «...devraient être considérés comme générateurs d'hypothèses et exigent leur confirmation ou, au contraire, leur réfutation, par des études randomisées contrôlées. " Mais quelles hypothèses sont générées par leurs résultats ? Telle est la question.

La première hypothèse pourrait être qu'un volume suffisant de liquide administré dès les premiers signes d'hypotension pourrait altérer le devenir d'une septicémie sévère. McIntyre et coll. ont quantifié le volume de liquide administré à leurs patients durant les six premières heures en 0-2 L, 2-4 L et $>4 \mathrm{~L}$. Mais nous ne savons pas pourquoi leurs patients ont reçu une quantité de liquide petite, intermédiaire ou grande. Etait-ce basé sur des résultats hémodynamiques ? Un protocole institutionnel? Une pratique courante ? Dans l'étude Rivers qui a servi de modèle aux auteurs, l'accent était mis non pas sur le volume ou le type de liquide utilisé, mais sur une approche thérapeutique aux objectifs déterminés, définie par une correction de l'hypotension, ou une saturation veineuse centrale en oxygène de $65 \%$. Cette approche est également préconisée dans le Lot de réanimation de septicémie de la Surviving Sepsis Campaign (http://ssc.sccm.org/).

Dans l'étude McIntyre, ${ }^{4}$ la quantité totale moyenne de liquide administré n'était que de 2,4 L, comparée à une moyenne de 5,0 L dans l'étude Rivers. ${ }^{6} \mathrm{La}$ différence pourrait-elle s'expliquer par l'application d'une approche thérapeutique aux objectifs déterminés dans la seconde étude ? Etant donné que McIntyre et coll. ${ }^{4}$ n'ont pas pu obtenir de détails quant au taux d'administration liquidienne ou sur le contexte global d'optimisation, leur étude ne peut éclairer le rapport entre le volume de liquide administré, l'atteinte de buts thérapeutiques, et le devenir des patients.

La deuxième hypothèse pourrait être qu'une combinaison de colloïdes et de cristalloïdes pourrait avoir un effet plus bénéfique sur les résultats que l'utilisation de cristalloïdes seuls. Toutefois, comme McIntyre et coll. ${ }^{4}$ l'admettent, une excellente étude génératrice d'hypothèse a déjà été menée: dans l'étude SAFE, une tendance vers une mortalité réduite a pu être observée dans un sous-groupe de septicémie sévère ayant reçu de l'albumine au lieu de solution saline. ${ }^{5}$ Il est malheureux que le Canadian Critical Care Trials Group n'ait pas choisi de tester cette hypothèse à l'aide d'une étude randomisée contrôlée ; peut-être le feront-ils maintenant ?

Nombre d'autres questions restent sans réponse. McIntyre et coll. ${ }^{4}$ ont exclu les patients ayant dével- 
oppé une septicémie sévère après les premières $24 \mathrm{~h}$ suivant l'admission aux soins intensifs ou après sept jours d'hospitalisation. Ceci pourrait constituer un sous-groupe important, particulièrement pour les septicémies se développant chez des patients post-chirurgicaux, qui ne constituait que $25 \%$ de leur étude. Le Canadian Critical Care Trials Group devrait examiner sa base de données afin de s'assurer quelle proportion cela représente du nombre total de patients souffrant de septicémie sévère, et s'ils présentent une différence significative dans le devenir. L'étude Rivers était basée sur une intervention précoce chez des patients admis en salle d'urgence. ${ }^{6}$ En fin de compte, il faut déterminer si les critères de l'étude Rivers (et le Lot de réanimation de septicémie de la Surviving Sepsis Campaign) sont aussi efficaces dans le traitement des patients qui développent une septicémie après les premières 24 h suivant l'admission aux soins intensifs et après sept jours d'hospitalisation.

Comment interpréter les observations des auteurs concernant le fait qu'un volume accru de liquide administré a été associé à une défaillance cardiovasculaire accrue mais à une défaillance rénale réduite ? On peut être tenté de conclure que «ce qui est mauvais pour le cœur est bon pour les reins ». Mais les deux événements, nommément la préservation de la fonction rénale et la défaillance cardiaque provoquée par une quantité plus importante de liquide, ont-ils été observés chez les mêmes patients ? Ou alors, est-il possible que les patients nécessitant davantage de liquide soient plus malades, souffrant a priori d'un syndrome de fuite capillaire et d'une dépression myocardique septique plus sévères ? Toutes ces hypothèses méritent d'être testées, mais il peut s'avérer difficile - ou même contraire à l'éthique - de concevoir une étude randomisée contrôlée.

L'observation la plus intrigante est peut-être le fait qu'une combinaison de colloïde et de cristalloïde a été associée à une défaillance rénale réduite. ${ }^{4}$ Cette observation pourrait-elle soutenir que la réanimation hémodynamique est plus réussie lorsqu'on ajoute du colloïde ? L'un des problèmes rencontrés lorsque les volumes liquidiens sont simplement comparés est que l'effet d'expansion du volume intravasculaire est très différent entre les liquides colloïde et cristalloïde. Dans l'étude SAFE, ${ }^{5}$ le volume de solution saline normale reconnu comme équivalent à de l'albumine $4 \%$ était compris entre 1,3 et $1,6: 1$. Dans les cercles d'anesthésiologie et de chirurgie, on compte d'ardents défenseurs de la restriction liquidienne peropératoire pendant une chirurgie gastro-intestinale qui basent leur conviction sur des données probantes démontrant de meilleurs devenirs postopératoires avec cette stra- tégie. ${ }^{8}$ Toutefois, lorsqu'on examine plus attentivement les études, on découvre qu'une approche avec "restriction liquidienne » substitue tout simplement le cristalloïde par du colloïde, diminuant ainsi le volume total administré tout en maintenant les bienfaits hémodynamiques. Ainsi, il est possible que dans l'étude dont il est ici question, les patients ont eu de meilleurs résultats rénaux avec une combinaison de colloïde et de cristalloïde, parce que le premier fournit une meilleure expansion de volume que le second lorsqu'il est administré seul.

Finalement, qu'avons-nous appris de cette étude observationnelle? Assurément, elle renforce les implications redoutables de la septicémie sévère. Plus d'un tiers des patients ont souffert de défaillance systémique de deux organes ou plus dans les premières 24 h suivant le début de la septicémie, la mortalité aux soins intensifs atteignait presque $35 \%$, la mortalité hospitalière était de plus de $45 \%$, et la moyenne de la durée du séjour aux soins intensifs et à l'hôpital était de six et quatorze jours respectivement.

Ce qui est plus important, comme les auteurs le soulignent, c'est que nous devons générer davantage de données probantes sur les conditions spécifiques de la prise en charge liquidienne précoce d'une septicémie sévère. Il semble très honorable de tester l'hypothèse selon laquelle, dans les cas de septicémie sévère, une réanimation précoce avec une combinaison liquidienne de colloïde et de cristalloïde donne de meilleurs résultats (et une préservation rénale) que le cristalloïde seul. Mais une telle étude se doit d'incorporer une approche thérapeutique aux objectifs déterminés - et l'atteinte de ces objectifs - en tant que critère premier de prise en charge, plutôt que le volume total de liquide utilisé.

\section{References}

l Anonymous. American College of Chest Physicians/ Society of Critical Care Medicine Consensus Conference: definitions for sepsis and organ failure and guidelines for the use of innovative therapies in sepsis. Crit Care Med 1992; 20: 864-74.

2 Angus DC, Linde-Zwirble WT, Lidicker J, Clermont G, Carcillo J, Pinsky MR. Epidemiology of severe sepsis in the United States: analysis of incidence, outcome, and associated costs of care. Crit Care Med 2001; 29: 1303-10.

3 Eichacker PQ Natanson C, Danner RL. Surviving sepsis--practice guidelines, marketing campaigns, and Eli Lilly. N Engl J Med 2006; 355: 1640-2.

4 McIntyre LA, Fergusson D, Cook DJ, et al.; Canadian Critical Care Trials Group. Quantity and type of fluid do not influence resuscitation of patients with early 
severe sepsis. Can J Anesth 2007; 54: 790-8.

5 Finfer S, Bellomo R, Boyce N, et al.; SAFE Study Investigators. A comparison of albumin and saline for fluid resuscitation in the intensive care unit. $\mathrm{N}$ Engl J Med 2004; 350: 2247-56.

6 Rivers E, Nguyen B, Havstad S, et al.; Early GoalDirected Therapy Collaborative Group. Early goaldirected therapy in the treatment of severe sepsis and septic shock. N Engl J Med 2001; 345: 1368-77.

7 Vincent JL, Moreno R, Takala J, et al. The SOFA (Sepsis-related Organ Failure Assessment) score to describe organ dysfunction/failure. On behalf of the Working Group on Sepsis-Related Problems of the European Society of Intensive Care Medicine. Intensive Care Med 1996; 22: 707-10.

8 Joshi GP. Intraoperative fluid restriction improves outcome after major elective gastrointestinal surgery. Anesth Analg 2005; 101: 601-5. 